\title{
Perioperative Serum 25-Hydroxyvitamin D Levels as a Predictor of Postoperative Opioid Use and Opioid Use Disorder: a Cohort Study
}

\author{
Yuhree Kim ${ }^{1,2}$, Fang Zhang ${ }^{7}$, Katherine Su ${ }^{1,2}$, Marc LaRochelle ${ }^{3}$, Matthew Callahan', \\ David Fisher' ${ }^{2}$ J. Frank Wharam?', and Maryam M. Asgari ${ }^{1,2}$ [D
}

'Department of Population Medicine, Harvard Medical School, 401 Park Drive \#401, Boston, MA, USA; ${ }^{2}$ Department of Dermatology, Massachusetts General Hospital, Harvard Medical School, 50 Staniford Street, Boston, MA, USA; ${ }^{3}$ Clinical Addiction Research and Education Unit, Section of General Internal Medicine, Department of Medicine, Boston University School of Medicine and Boston Medical Center, Boston, MA, USA.

IMPORTANCE: Vitamin D deficiency is associated with chronic pain syndromes and higher opioid use among cancer patients, but its association with opioid use among opioid-naïve subjects following a major surgical procedure with acute pain has not been explored.

OBJECTIVE: To determine the association between serum 25-hydroxyvitamin D $(25(\mathrm{OH}) \mathrm{D})$ levels, opioid use, and opioid use disorder.

METHODS: We identified commercially insured subjects aged 18-64 years with available perioperative serum 25hydroxyvitamin D (25D) levels who underwent one of nine major surgical procedures in 2000-2014. Primary outcomes were dose and duration of opioid use measured using pharmacy claims. Secondary outcome was opioid use disorder captured using diagnosis codes. Multivariable negative binomial models with generalized estimating equations were performed examining the association between 25D levels and postoperative opioid use measures, adjusting for age, sex, race/ethnicity, Charlson score, education, income, latitude, and season of blood draw. Adjusted Cox regression was used to examine the association with opioid use disorder.

RESULTS: Among 5446 subjects, serum 25(OH)D was sufficient ( $\geq 20 \mathrm{ng} / \mathrm{mL}$ ) among 4349 (79.9\%) subjects, whereas 837 (15.4\%) had insufficient ( 12 to $<20 \mathrm{ng} / \mathrm{mL}$ ) and $260(4.8 \%)$ had deficient $(<12 \mathrm{ng} / \mathrm{mL})$ levels. On multivariable analysis, as compared with subjects with sufficient 25(OH)D levels, subjects with deficient $25(\mathrm{OH}) \mathrm{D}$ levels had 1.7 more days $(95 \% \mathrm{CI} 0.76,2.58)$ of opioid use per year and had 98.7 higher morphine milligram equivalent dose $(95 \%$ CI 55.7, 141.8) per year. Among 11,713 study cohort, subjects with deficient 25(OH)D levels were more likely to be diagnosed with opioid use disorders (HR 2.41; 95\% CI 1.05, 5.52).

CONCLUSION: Patients undergoing common surgical procedures with deficient 25D levels are more likely to have higher opioid use and an increased risk of opioid use disorder compared to those with sufficient levels.

Electronic supplementary material The online version of this article (https://doi.org/10.1007/s11606-020-06001-y) contains supplementary material, which is available to authorized users.

Received October 8, 2019

Accepted June 17, 2020

Published online June 24, 2020
Serum 25D levels may serve as a biomarker to identify subjects at increased risk of opioid misuse.

KEY WORDS: vitamin D; 25(OH)D; chronic opioid use; opioid use disorder; opioid-related disorders/epidemiology.

J Gen Intern Med 35(9):2545-52 DOI: $10.1007 / \mathrm{s} 11606-020-06001-\mathrm{y}$ (c) Society of General Internal Medicine 2020

\section{INTRODUCTION}

Harms associated with opioid use are a national crisis and have been declared by the US President as a "public health emergency." A growing body of research has demonstrated the devastating public health impact of non-medical opioid use, opioid use disorder, and overdose deaths. ${ }^{2-5}$ Opioid-related deaths increased by more than fivefold between 1999 and 2017. ${ }^{6}$ Adverse health outcomes related to prescription opioids impose a substantial burden not only on patients but also on the health care system. Economic costs for opioidrelated health care, substance use treatment, and lost productivity were estimated at $\$ 78.5$ billion in $2013 .^{7}$

Many patients experience their first exposure to opioids after surgery, and previous studies reported that prescription opioids during and after surgery might trigger long-term use in patients regardless of previous opioid exposure. ${ }^{8-10}$ Among patients treated with opioid analgesics, several factors have been associated with the development of opioid use disorder, ${ }^{11-14}$ including opioid duration and dosage. ${ }^{15}$ Clinical tools to identify individuals at high risk to progress to long-term opioid use and opioid use disorder could assist provider decision-making around opioid prescribing. There are currently no biomarkers that can help identify subjects who are at increased risk of long-term opioid use and opioid use disorder when short-term opioid use is indicated, such as for short-term pain control following a major surgical procedure.

Vitamin D plays a critical role in a variety of biologic processes including musculoskeletal health. ${ }^{16,17}$ In previous studies, vitamin D deficiency has been correlated with both 
acute pain including postoperative and post-traumatic pain, and chronic pain, including non-specific musculoskeletal pain, ${ }^{18}$ widespread chronic pain, ${ }^{19}$ postoperative pain, ${ }^{20}$ lower back pain, ${ }^{21}$ fibromyalgia, ${ }^{22}$ and headache. ${ }^{23}$ Low serum vitamin D levels have been associated with increased pain perception in chronic pain patients, ${ }^{24,25}$ as well as with higher opioid doses in patients with chronic pain. ${ }^{26,}{ }^{27}$ Despite a possible relationship between vitamin $\mathrm{D}$ and pain as well as the substantial public health importance of both vitamin D deficiency and chronic opioid use, no studies, to our knowledge, have examined whether vitamin D deficiency is associated with opioid misuse. We hypothesized that subjects with low serum vitamin D levels would be more likely to use opioids at a higher dose and for a longer period and become opioid-dependent following surgery. Thus, the aim of the study was to examine the association between perioperative serum vitamin D levels and the duration and dose of opioid use and opioid use disorder among commercially insured subjects following commonly performed major surgeries.

\section{METHODS}

\section{Setting and Population}

This retrospective cohort study was conducted using the Optum's de-identified Clinformatics ${ }^{\circledR}$ Data Mart Database, which contains inpatient, outpatient, and pharmacy claims for approximately 50 million members in all 50 states. We identified 14,356 subjects who underwent one of nine surgical procedures (total knee arthroplasty, total hip arthroplasty, laparoscopic and open cholecystectomy, laparoscopic and open appendectomy, Cesarean section, functional endoscopic sinus surgery, and transurethral prostate resection) between May 2000 and December 2014 and had vitamin D levels measured within 3 months perioperatively (Fig. 1). The nine surgical procedures were selected based on a previous opioid use study that examined risk factors for chronic opioid use in the postoperative period. ${ }^{11}$ Surgeries were identified using Current Procedural Terminology codes (eTable 1). If individuals underwent more than one eligible procedure during the study period, we included only the first as the index procedure ("index date"). Subjects were excluded if they (1) were not continuously enrolled for at least 6 months prior to index $(n=$ 1656), (2) were aged less than 18 or greater than 64 years at index ( $n=939)$, and (3) had missing demographic or followup data $(n=10)$, leaving 11,751 individuals. We then constructed two different cohorts based on the outcome measure to be analyzed (Fig. 1). To examine the number of days supply of opioids and a total dosage of opioid use among opioid-naïve subjects, our sample was restricted to patients who initiated opioids in the early postoperative phase (days 0 through 60 ). We required that initiators have a minimum of 6 months of preoperative enrollment, at least 60 days postoperative enrollment, no opioid dispensings during the 6 months before surgery, and at least 1 opioid prescription within the 60 days following surgery. To examine incident opioid use disorder, we excluded individuals who had evidence of this disorder in the 6 months before surgery. Participants were censored at (1) dis-enrollment from the health plan; (2) 5 years after the index surgery; (3) age of 65 years (Medicare eligible); or (4) study end date (December 31, 2014), whichever occurred first. This study was approved by the Harvard Pilgrim Health Care Institutional Review Board.

\section{Exposure Variable}

Serum 25(OH)D levels were available on 100,057 patients out of the 1,863,452 undergoing the procedures of interest (5.4\%), and 14,356 had $25(\mathrm{OH}) \mathrm{D}$ levels measured in the 3 months before or after the index surgery. When an individual had multiple measurements of $25(\mathrm{OH}) \mathrm{D}$ within the perioperative period, we used the $25(\mathrm{OH}) \mathrm{D}$ level that was measured closest to the date of surgery. We defined 25(OH)D levels $<12 \mathrm{ng} / \mathrm{mL}$ as deficient, 12 to $<20 \mathrm{ng} / \mathrm{mL}$ as insufficient, and $\geq 20 \mathrm{ng} / \mathrm{mL}$ as sufficient, per Endocrine Society Global Consensus Recommendations. ${ }^{28}$

\section{Outcome Variable}

Dispensings of oral or transdermal opioids prescription opioids were identified from Optum pharmacy claims data, as previously described. ${ }^{29}$ Opioids included codeine, fentanyl, hydrocodone, hydromorphone, levorphanol, meperidine, methadone, morphine, oxycodone, oxymorphone, propoxyphene, tapentadol, and tramadol. The active ingredient, milligram strength, and formulation type were determined by matching to National Drug Code numbers. The outcomes of interest were (1) opioid use, examined by both number of days of opioids and total morphine equivalent dose (MED) during the study period (eFigure 1) and (2) opioid use disorder (eFigure 2).

Number of days of opioids was calculated by adding the number of opioid days supplied from postoperative day 61 to the end of a member's eligible follow-up time, accounting for early refills and any overlaps. We did not include opioid use during the 60-day postoperative period because opioid use during this time is related to acute pain rather than chronic post-surgical pain. ${ }^{30}$ When a refill for an opioid prescription occurred before the calculated prescribed end date, the overlapping portions of the prescription were assumed to be taken concurrently. When an individual received two dispensings the same day, the dispensing with the greater number of days supply was used for the calculation.

We calculated MED for a single opioid dispensing by multiplying the quantity of each prescription by the strength (milligrams of opioid per unit dispensed) of the prescription opioid, which was then multiplied by the conversion factor for morphine equivalents using standard conversion tables mapping the amount of morphine equivalent to a given type of drug and dose. ${ }^{31}$ Daily morphine milligram equivalents (MME) were calculated by dividing the total MED per 


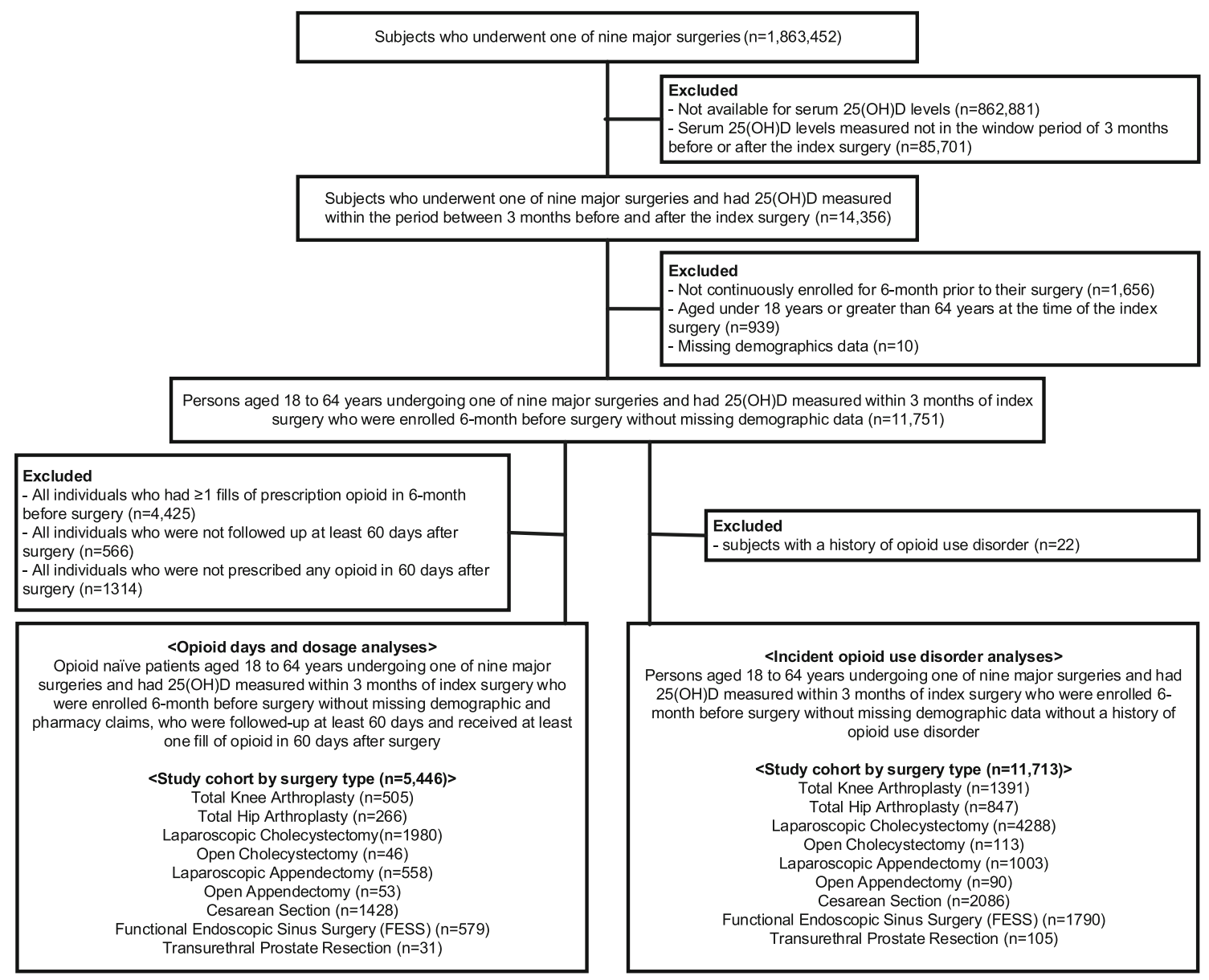

Figure 1 Flow diagram of inclusions and exclusions of study cohort for opioid use and opioid use disorder analyses.

dispensing by the total of days supply assuming subjects took the maximum dose prescribed per day, as previously described. ${ }^{29}$ If a subject had opioids available from multiple dispensings on the same day, morphine equivalents were summed for that day for the calculation of daily MME.

As an additional outcome measure, we identified opioid use disorders using ICD-9 (International Classification of Diseases, ninth revision) codes (opioid abuse, 305.50-305.52; opioid dependence, 304.00-304.02; and combinations of opioid type drug with any other drug dependence, 304.70304.72). ${ }^{32}$ Opioid use disorder was defined as the presence of at least one of these ICD codes after the index date.

\section{Covariates}

Demographic variables included age, sex, race/ethnicity, and socioeconomic variables including education and income levels. To define proxy demographic measures including race/ethnicity, neighborhood income, and education level, we used validated categorical variables termed the Index of Concentration at the Extremes (ICE) that captures the concentration of poverty and racial segregation at the census tract-level, as previously described. ${ }^{33,} 34$ To account for variation in vitamin D levels, we examined latitude of residence based on patient's residential state as previously described. ${ }^{27} \mathrm{We}$ also assessed season of blood draw (Nov 1-Apr 30 vs. May 1-Oct 31) to account for seasonal variation of serum vitamin D levels. ${ }^{35} \mathrm{~A}$ Charlson score was calculated using the STATA $®$ (StataCorp, College Station, TX) module. A history of obesity (ICD-9-CM 278, and 278.01-03), bone fracture (ICD-9-CM 733.10-19, 733.93-98, and 800-829), and osteoporosis (ICD-9-CM 733, 733.01-03, and 733.09) and a calculated Charlson score were captured using ICD-9 diagnosis codes from inpatient, emergency department (ED), and outpatient claims during the 6month pre-index period. We required that patients have at least one inpatient diagnosis or at least two outpatient or ED diagnoses to qualify as having given comorbidity.

\section{Statistical Analysis}

We used the one-way ANOVA test to assess univariable comparisons across the vitamin D groups for the continuous variable (age), while the chi-squared test was used to assess the remaining categorical variables. Multivariable negative binomial models with generalized estimating equations (GEE) were performed to calculate adjusted risk ratios (aRRs) for number of days covered per year and MED per year, accounting for follow-up time and potential confounders. We then analyzed the marginal effects of the GEE model to calculate the differences in days covered by opioid and total 
MED across the vitamin D group. We adjusted for possible confounding variables chosen a priori based on previous studies, ${ }^{36}$ including age, sex, race/ethnicity, education levels, income levels, season of blood draw, latitude of residence, Charlson score, history of obesity, history of bone fracture, and history of osteoporosis. Multivariable Cox regression models were used to calculate adjusted hazard ratios (HRs), including opioid prescriptions in the 6 months prior to the index, for the risk of incident opioid use disorder. Statistical analyses were performed with STATA, version 15.0 (StataCorp, College Station, TX), and SAS, version 9.4 (SAS Institute); all tests were two-sided.

\section{RESULTS}

\section{Vitamin D Levels and Opioid Use}

The mean age of the 5446 study subjects was 44 years, and $80.1 \%$ were female (Table 1$)$. The median $25(\mathrm{OH}) \mathrm{D}$ level was $28.7 \mathrm{ng} / \mathrm{mL}$ (interquartile range [IQR] 21.5-36.9 ng/mL). The majority of the subjects $(n=4349,79.8 \%)$ had $25(\mathrm{OH}) \mathrm{D}$ levels in the sufficient range, with the remainder measuring either in the insufficient $(n=837,15.4 \%)$ or in the deficient $(n=260,4.8 \%)$ range. A total of $43 \%$ of subjects $(n=2328)$ had $25(\mathrm{OH}) \mathrm{D}$ levels measured before or on the day of the index surgery while the remainder $(n=3118,57.2 \%)$ had testing after the index surgery. The median follow-up time of the study cohort was 1.3 (IQR 0.6-2.3) years (range 0.2 to 5.0 years). The median follow-up time of subjects with sufficient, insufficient, and deficient 25(OH)D levels was 1.2 (IQR 0.6-2.3), 1.3 (IQR 0.7-2.4), and 1.5 (IQR 0.8-2.7) years, respectively $(P<0.001)$. When compared with subjects in the insufficient or sufficient group, those in the deficient group were more likely to be non-Hispanic Black ( $8.1 \%$ vs. $3.5 \%$ vs. $2.3 \%$, respectively), low income ( $13.5 \%$ vs. $11.6 \%$ vs. $7.0 \%$, respectively), and less educated ( $33.5 \%$ vs. $27.2 \%$ vs. $19.4 \%$, respectively); have $25(\mathrm{OH}) \mathrm{D}$ measured in winter months ( $65.8 \%$ vs. $52.8 \%$ vs. $46.3 \%$, respectively); and were more obese with a previous diagnosis of obesity $(17.3 \%$ vs. $13.7 \%$ vs. $9.8 \%$, respectively) (all $P<0.05$ ). The Charlson score and latitude of residence did not differ by serum 25(OH)D categories.

The mean days covered by opioid use of subjects with sufficient, insufficient, and deficient $25(\mathrm{OH}) \mathrm{D}$ levels were in $4.8,4.8$, and 7.0 days per year, respectively (Table 2). After adjusting for potential confounders, subjects with deficient $25(\mathrm{OH}) \mathrm{D}$ levels had more days covered by opioid use (adjusted risk ratio [aRR] 1.34; 95\% CI 1.17, 1.55; $p<0.001$ ) while those with insufficient $25(\mathrm{OH}) \mathrm{D}$ levels did not reach statistical significance (aRR 1.02; 95\% CI 0.93, 1.11; $P=0.709$ ) when compared to those with sufficient 25(OH)D levels (Fig. 2a). Subjects with deficient 25(OH)D levels had 1.67 more days (95\% CI 0.76-2.58) of opioid use per year than those with sufficient $25(\mathrm{OH}) \mathrm{D}$ levels while those with insufficient
25(OH)D levels had similar opioid days when compared with sufficient 25(OH)D levels (Fig. 2b).

The mean total MED values of subjects with sufficient, insufficient, and deficient 25(OH)D level were 211.0, 279.1, and 394.9 per year, respectively (Table 2). On multivariable analyses, subjects with deficient 25(OH)D levels showed higher doses of opioid use when compared with sufficient 25(OH)D levels (aRR 1.41; 95\% CI 1.24, 1.60; $p<0.001$ ) while those with insufficient $25(\mathrm{OH}) \mathrm{D}$ levels did not reach statistical significance (aRR 1.07; 95\% CI 0.99, 1.15; $P=$ 0.09 ) (Fig. 3a). In specific, subjects with deficient 25(OH)D levels had 98.7 MED (95\% CI 55.7, 141.8) per year more than those with sufficient $25(\mathrm{OH}) \mathrm{D}$ levels while those with insufficient 25(OH)D levels had similar MED when compared with sufficient 25(OH)D levels (Fig. 3b).

Given that hip and knee replacements may be performed to relieve chronic pain, sensitivity analyses were performed excluding those who underwent total knee and hip arthroplasties $(n=771)$. Subjects with deficient 25(OH)D levels had more days covered by opioid use (aRR 1.23; 95\% CI 1.06, 1.44; $P=0.007)$ when compared to those with sufficient $25(\mathrm{OH}) \mathrm{D}$ levels.

\section{Vitamin D Levels and Incident Opioid Use Disorder}

A total of 11,713 subjects without a history of opioid use disorder prior to the index surgery were included in the analysis (eFigure 2). The baseline characteristics of the study cohort for incident opioid use disorder analyses are summarized in eTable 2. During the follow-up period, incident opioid use disorder was coded for 61 subjects $(0.5 \%$; sufficient 41 [0.4\%] vs. insufficient 13 [0.7\%] vs. deficient 7 [1.2\%]) (Table 3). When accounting for potential confounders and history of opioid use, subjects with deficient $25(\mathrm{OH}) \mathrm{D}$ levels had increased risk of opioid use disorder (aHR 2.41; 95\% CI $1.05,5.52 ; p=0.038$ ), while opioid use disorder among those with insufficient $25(\mathrm{OH}) \mathrm{D}$ levels did not reach statistical significance (aHR 1.73; 95\% CI 0.92-3.28; $p=0.09$, Fig. 4) when compared to those with sufficient $25(\mathrm{OH}) \mathrm{D}$ levels.

\section{DISCUSSION}

We found that deficient vitamin D levels among opioid-naïve subjects were associated with increased number of days of postoperative opioid use and higher total opioid dose when compared to those with sufficient vitamin D levels. The association remained significant after adjusting for potential confounding factors including demographics and comorbidities. Furthermore, vitamin D deficiency was associated with incident opioid use disorder. Our findings need to be interpreted with caution and do not imply that subjects with normal perioperative vitamin D levels are not at risk for opioid dependence. Many postoperative pain management options, 
Table 1 Baseline Characteristics Based on Serum Vitamin D Level

\begin{tabular}{|c|c|c|c|c|c|}
\hline \multirow[t]{2}{*}{ Characteristic } & \multirow{2}{*}{$\begin{array}{l}\text { Total } \\
N=5446\end{array}$} & \multirow{2}{*}{$\begin{array}{l}\text { Deficient } \\
N=260\end{array}$} & \multirow{2}{*}{$\begin{array}{l}\text { Insufficient } \\
N=837\end{array}$} & \multirow{2}{*}{$\begin{array}{l}\text { Normal } \\
N=4349\end{array}$} & \multirow[t]{2}{*}{$P$} \\
\hline & & & & & \\
\hline Age, year, mean $( \pm \mathrm{SD})$ & $44.3( \pm 12.0)$ & $43.5( \pm 11.8)$ & $42.8( \pm 11.3)$ & $44.7( \pm 12.1)$ & $<0.001(F$-value 8.67$)$ \\
\hline Gender & & & & & 0.022 \\
\hline Female & $4364(80.1)$ & $198(76.2)$ & $649(77.5)$ & $3517(80.9)$ & \\
\hline Male & 1082 (19.9) & $62(23.8)$ & $188(22.5)$ & $832(19.1)$ & \\
\hline $\operatorname{Race}^{\mathrm{a}}$ & $1002(19.3)$ & $02(23.0)$ & $100(22.0)$ & $032(19.1)$ & $<0.001$ \\
\hline Quintile 1 & $151(2.8)$ & $21(8.1)$ & $29(3.5)$ & $101(2.3)$ & \\
\hline Quintile 2 & $209(3.8)$ & $17(6.5)$ & $46(5.5)$ & $146(3.4)$ & \\
\hline Quintile 3 & $512(9.4)$ & $39(15.0)$ & 97 (11.6) & $376(8.6)$ & \\
\hline Quintile 4 & $1183(21.7)$ & $67(25.8)$ & $205(24.5)$ & $911(20.9)$ & \\
\hline Quintile 5 & $3391(62.3)$ & $116(44.6)$ & $460(55.0)$ & $2815(64.7)$ & \\
\hline Household income ${ }^{\mathrm{b}}$ & 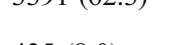 & ) 10 (ד. & (3) & 2010 (0ד. & $<0.001$ \\
\hline Quintile 1 & $435(8.0)$ & $35(13.5)$ & $97(11.6)$ & $303(7.0)$ & \\
\hline Quintile 2 & $767(14.1)$ & $65(25.0)$ & $135(16.1)$ & $567(13.0)$ & \\
\hline Quintile 3 & $944(17.3)$ & $46(17.7)$ & $146(17.4)$ & $752(17.3)$ & \\
\hline Quintile 4 & $1170(21.5)$ & $46(17.7)$ & $170(20.3)$ & $954(21.9)$ & \\
\hline Quintile 5 & $2130(39.1)$ & $68(26.1)$ & $289(34.5)$ & $1773(40.8)$ & \\
\hline Education $^{\mathrm{c}}$ & & & & & $<0.001$ \\
\hline Quintile 1 & $1158(21.3)$ & $87(33.5)$ & $228(27.2)$ & $843(19.4)$ & \\
\hline Quintile 2 & $900(16.5)$ & $53(20.4)$ & $154(18.4)$ & $693(15.9)$ & \\
\hline Quintile 3 & $841(15.4)$ & $31(11.9)$ & $133(15.9)$ & $677(15.6)$ & \\
\hline Quintile 4 & $1131(20.8)$ & $49(18.8)$ & $145(17.3)$ & $937(21.5)$ & \\
\hline Quintile 5 & $1416(26.0)$ & $40(15.4)$ & $177(21.2)$ & $1199(27.6)$ & \\
\hline Season of blood draw & & & & & $<0.001$ \\
\hline November 1-April 30 & $2625(48.2)$ & $171(65.8)$ & $442(52.8)$ & $2012(46.3)$ & \\
\hline May $1-$ October 31 & $2821(51.8)$ & $89(34.2)$ & $395(47.2)$ & $2337(53.7)$ & \\
\hline Latitude of residence $(n=5442)$ & & & & & 0.519 \\
\hline$>37^{\circ}$ North & $2301(42.3)$ & $117(45.0)$ & $362(43.2)$ & $1822(41.9)$ & \\
\hline$\leq 37^{\circ}$ North & $3140(57.7)$ & $143(55.0)$ & $475(56.8)$ & $2522(58.1)$ & \\
\hline Charlson score & & & & & 0.299 \\
\hline 0 & 3727 (68.4) & $169(65.0)$ & $575(68.7)$ & $2983(68.6)$ & \\
\hline $1-2$ & $1500(27.6)$ & $75(28.8)$ & $224(26.8)$ & $1201(27.6)$ & \\
\hline$\geq 3$ & $219(4.0)$ & $16(6.2)$ & $38(4.5)$ & $165(3.8)$ & \\
\hline Obesity & $585(10.7)$ & $45(17.3)$ & $115(13.7)$ & $425(9.8)$ & $<0.001$ \\
\hline Bone fracture & $40(0.7)$ & $5(1.9)$ & $6(0.7)$ & $29(0.7)$ & 0.07 \\
\hline Osteoporosis & $51(0.9)$ & $3(1.1)$ & $2(0.2)$ & $46(1.1)$ & 0.074 \\
\hline Surgical procedures & & & & & $<0.001$ \\
\hline Total knee arthroplasty & $505(9.3)$ & $21(8.1)$ & $56(6.7)$ & $428(9.8)$ & \\
\hline Total hip arthroplasty & $266(4.9)$ & $5(1.9)$ & $26(3.1)$ & $235(5.4)$ & \\
\hline Laparoscopic cholecystectomy & $1980(36.4)$ & $140(53.9)$ & 351 (41.9) & $1489(34.2)$ & \\
\hline Open cholecystectomy & $46(0.8)$ & $5(1.9)$ & $9(1.1)$ & $32(0.7)$ & \\
\hline Laparoscopic appendectomy & $558(10.2)$ & $24(9.2)$ & $96(11.5)$ & $438(10.1)$ & \\
\hline Open appendectomy & $53(1.0)$ & $1(0.4)$ & $11(1.3)$ & $41(0.9)$ & \\
\hline Cesarean section & $1428(26.2)$ & $36(13.8)$ & $186(22.2)$ & $1206(27.7)$ & \\
\hline Functional endoscopic sinus surgery (fess) & $579(10.6)$ & $26(10.0)$ & $95(11.4)$ & $458(10.5)$ & \\
\hline Transurethral prostate resection & $31(0.6)$ & $2(0.8)$ & $7(0.8)$ & $22(0.5)$ & \\
\hline Follow-up time, years, median (IQR) & $1.3(0.6-2.3)$ & $1.5(0.8-2.7)$ & $1.3(0.7-2.4)$ & $1.2(0.6-2.3)$ & $<0.001$ \\
\hline
\end{tabular}

${ }^{a}$ Q1: least concentrated non-Hispanic Whites; Q5: highly concentrated non-Hispanic Whites

${ }^{b}$ Q1: least concentrated neighborhood with high income; Q5: highly concentrated neighborhood with high income

${ }^{c} Q 1$ : least concentrated neighborhood with high education; Q5: highly concentrated neighborhood with high education

including non-opioid medications, are available and should be carefully considered by the prescribing provider.

While low vitamin D levels have been associated with the amount and duration of short-term opioid use in palliative cancer patients, previous studies have not evaluated the association with vitamin D levels and opioid use with longer

Table 2 Mean Days Supply of Opioid Use and Morphine Milligram Equivalent Dosage per Year

\begin{tabular}{llll}
\hline \hline $\begin{array}{l}\text { Vitamin D } \\
\text { levels }\end{array}$ & $\boldsymbol{N}$ & $\begin{array}{l}\text { Mean days } \\
\text { supply of opioids } \\
\text { per year }\end{array}$ & $\begin{array}{l}\text { Mean morphine } \\
\text { milligram equivalent } \\
\text { dosage per year }\end{array}$ \\
\hline$<12 \mathrm{ng} / \mathrm{mL}$ & 260 & 7.01 & 394.91 \\
$12-20 \mathrm{ng} /$ & 837 & 4.84 & 279.07 \\
$\mathrm{~mL}$ & & & 211.04 \\
$\geq 20 \mathrm{ng} / \mathrm{mL}$ & 4349 & 4.77 & 230.27 \\
\hline Total & 5446 & 4.89 &
\end{tabular}

follow-up. ${ }^{26,27}$ We assessed opioid use over the longer term by identifying subjects in a large commercially insured cohort, for whom vitamin D levels were measured perioperatively, and reviewing their pharmacy claims data prospectively during the study period. As such, we were able to analyze the duration and doses of opioid use during long-term follow-up, which have been associated with development of opioid use disorder. Future studies should examine nuances of opioid use in the postoperative period including the impact of the initial dose of opioid use as a predictor of long-term opioid use.

Vitamin D levels have been associated with a wide range of health conditions such as infectious, autoimmune, neuromuscular disorders, as well as pain syndrome. ${ }^{18,20,22,23,37-39}$ From an interventional standpoint, vitamin D supplementation reduced musculoskeletal pain in some studies ${ }^{40-42}$ and decreased opioid doses. ${ }^{43}$ While the underlying mechanism 


\section{a}

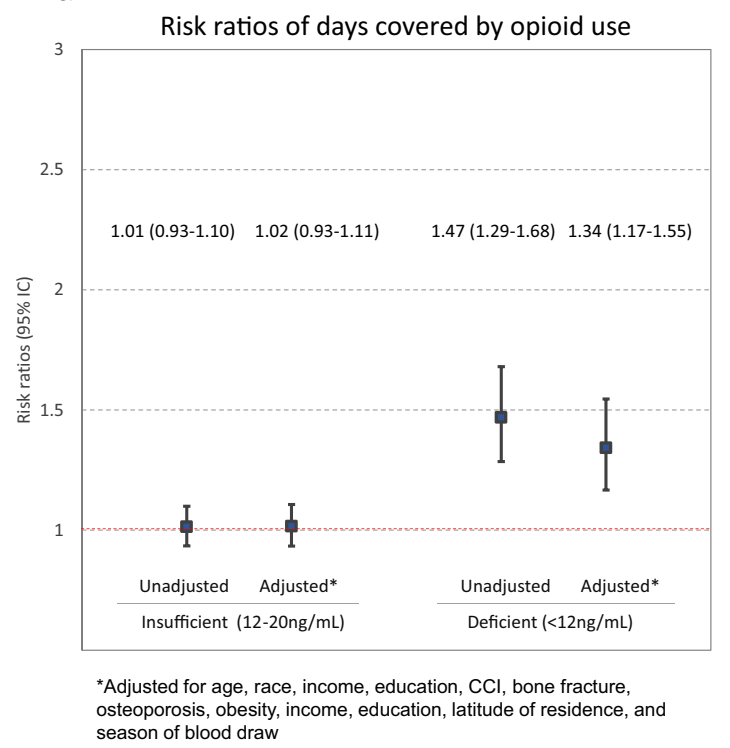

b

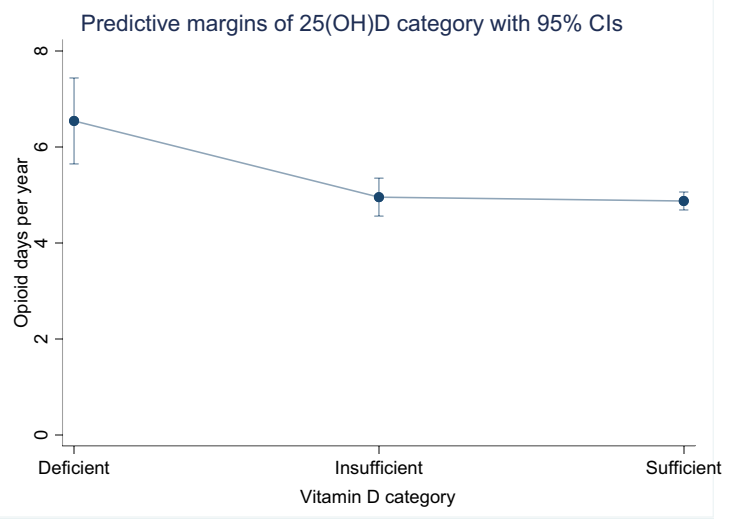

Figure 2 a Unadjusted and adjusted risk ratios of the number of days covered by opioid use stratified by insufficient vitamin D levels (12-20 ng/ $\mathrm{mL})$ and deficient vitamin $D$ levels $(<12 \mathrm{ng} / \mathrm{mL})$. b Predictive margins of number of days covered by opioid use per year by vitamin $D$ levels.

linking vitamin $\mathrm{D}$ to the development and continuation of pain remains unclear, the neuro-immunomodulatory action of vitamin $\mathrm{D}$ has been proposed to be a probable mechanism that can lead to pain sensitization. ${ }^{43}$ In fact, low vitamin D levels were correlated with higher pain intensity and increased central sensitivity in patients with chronic pain. ${ }^{25,}{ }^{44}$ In rodents, vitamin $\mathrm{D}$ deficiency promoted nociceptor hyperinnervation of skeletal muscles, contributing to muscular hypersensitivity and pain. ${ }^{45}$ Fell et al. reported that chronic UV exposure resulted in strong analgesic effects, increased pain thresholds, as well as opioid tolerance/physical dependence mediated by induction of an endogenous opioid-like substance $(\beta$ endorphins) in the skin. ${ }^{46}$ Given that vitamin D is synthesized in the skin through the pathway that needs UV light, further studies to evaluate the physiologic mechanism linking UV exposure, vitamin D levels, and opioid addiction may be warranted.

The current study has several limitations. The ICD-9 codes we used to define opioid use disorder have not yet been validated based on DSM-V diagnostic criteria and may present physiologic dependence resulting from long-term opioid use. Also, we used opioid dispensing in pharmacy claims data to analyze total duration and dosage which could both overreport opioid use (among subjects who fill prescriptions but do not take them) and under-report opioid use (among subjects who use opioids from other sources), but these measurement

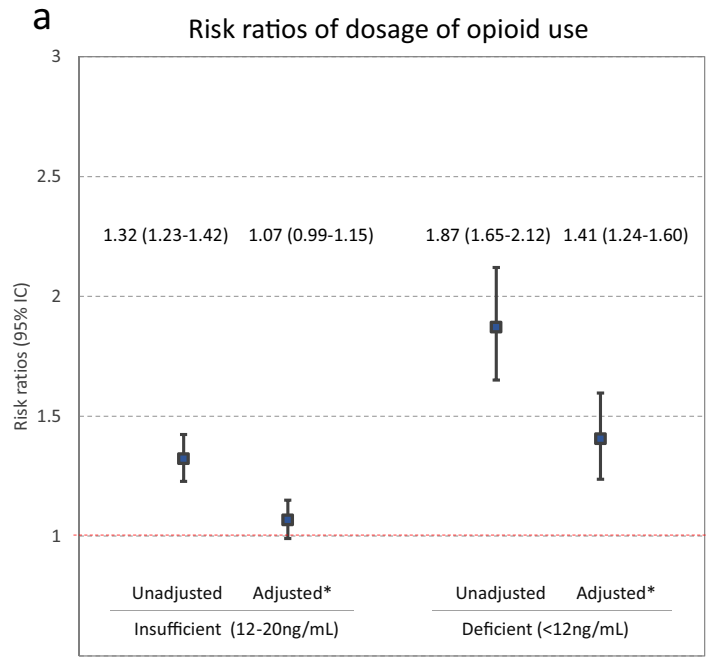

*Adjusted for age, race, income, education, $\mathrm{CCl}$, bone fracture osteoporosis, obesity, income, education, latitude of residence, and season of blood draw b

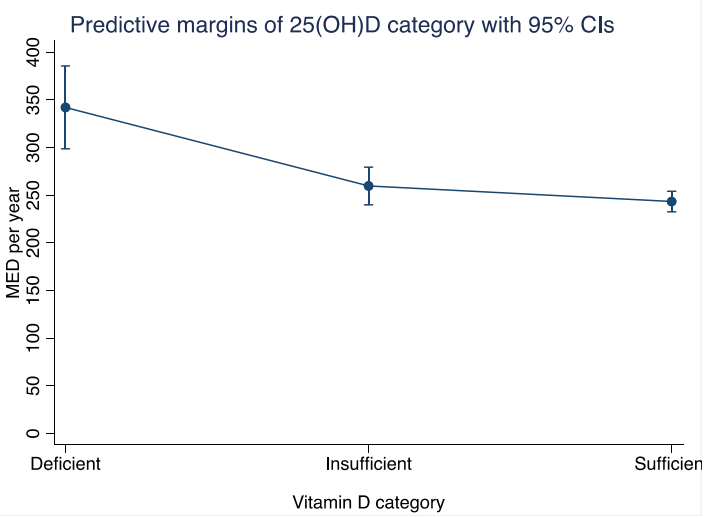

Figure 3 a Unadjusted and adjusted risk ratios of dosage of opioid use stratified by insufficient vitamin $\mathrm{D}$ levels (12-20 $\mathrm{ng} / \mathrm{mL})$ and deficient vitamin D levels $(<12 \mathrm{ng} / \mathrm{mL})$. b Predictive margins of total morphine equivalent dose per year by vitamin $D$ levels. 
Table 3 The Number of Subjects with Incident Opioid Use Disorder Stratified by Vitamin D Levels

\begin{tabular}{lll}
\hline \hline Vitamin D levels & $\boldsymbol{N}$ & Opioid use disorder $(\boldsymbol{n}[\%])$ \\
\hline$<12 \mathrm{ng} / \mathrm{mL}$ & 601 & $7(1.16 \%)$ \\
$12-20 \mathrm{ng} / \mathrm{mL}$ & 1813 & $13(0.72 \%)$ \\
$\geq 20 \mathrm{ng} / \mathrm{mL}$ & 9299 & $41(0.44 \%)$ \\
Total & 11,713 & $61(0.5 \%)$ \\
\hline
\end{tabular}

errors are likely to bias toward the null. Second, we do not know the indication for vitamin $\mathrm{D}$ measurement around the time of surgery, and we were unable to follow-up on changes in vitamin D levels and vitamin D supplementations in each subject over the study period. Third, we were unable to capture detailed clinical data and account for all relevant variables, such as body mass index (BMI), and could not eliminate residual confounding. To attempt to address this limitation, we captured the diagnosis of obesity using ICD codes, but obesity may not always be coded and, therefore, may be an incomplete surrogate for BMI. Finally, while this commercial database covers a broad US geographical representation, the study population does not capture uninsured individuals or older Medicare enrollees. The exclusion of such subjects may have led to an underestimation of the true burden of vitamin D deficiency and opioid misuse. Future studies among older individuals can help assess the generalizability of our findings.

\section{CONCLUSION}

In summary, our results suggest that individuals with deficient perioperative serum 25(OH)D levels are at increased risk for an increase in opioid duration and dose and incident opioid use disorder. These findings highlight a potential biomarker that

\section{Hazard ratios of opioid use disorder}

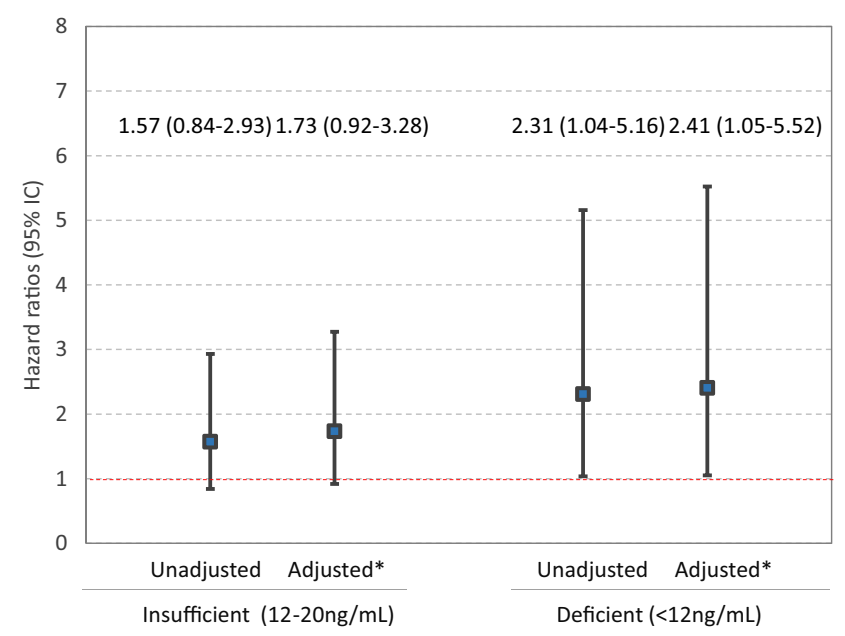

*Adjusted for age, race, income, education, $\mathrm{CCl}$, bone fracture, osteoporosis, obesity, income, education, latitude of residence, season of blood draw, and opioid prescriptions in the 6 months prior to the index

Figure 4 Unadjusted and adjusted hazard ratios of incident opioid use disorder stratified by insufficient vitamin $D$ levels $(12-20 \mathrm{ng} /$ $\mathrm{mL})$ and deficient vitamin D levels $(<12 \mathrm{ng} / \mathrm{mL})$. can be used to predict individuals at increased risk for opioid use disorder. Future studies are needed to assess whether vitamin D supplementations in the perioperative period can reduce the risk of long-term opioid use and incident opioid use disorder.

Corresponding Author: Maryam M. Asgari, Department of Population Medicine, Harvard Medical School, 401 Park Drive \#401, Boston, MA 02215, USA (e-mail: masgari@partners.org).

Funding Information M.M.A. is supported by the National Institute of Arthritis and Musculoskeletal and Skin Diseases (K24 AR069760). D.E.F. acknowledges grant support from NIH (5P01 CA163222, 1R01CA222871, and 5R01 AR043369-22), the Melanoma Research Alliance, and the Dr. Miriam and Sheldon G. Adelson Medical Research Foundation.

\section{Compliance with Ethical Standards:}

This study was approved by the Harvard Pilgrim Health Care Institutional Review Board.

Conflict of Interest: Dr. Fisher has a financial interest in Soltego, Inc., a company developing SIK inhibitors for topical skin darkening treatments that might be used for a broad set of human applications. Dr. Fisher's interests were reviewed and are managed by Massachusetts General Hospital and Partners Healthcare in accordance with their conflict of interest policies.

\section{REFERENCES}

1. President Donald J. Trump is Taking Action on Drug Addiction and the Opioid Crisis. The White House. https://www.whitehouse.gov/briefingsstatements/president-donald-j-trump-taking-action-drug-addiction-opioid-crisis/. .

2. Larochelle MR, Liebschutz JM, Zhang F, Ross-Degnan D, Wharam JF. Opioid Prescribing After Nonfatal Overdose and Association With Repeated Overdose: A Cohort Study. Ann Intern Med. 2016;164(1):1-9. doi:https://doi.org/10.7326/M15-0038

3. Volkow ND, McLellan TA. Curtailing diversion and abuse of opioid analgesics without jeopardizing pain treatment. JAMA. 2011;305(13):1346-7. doi:https://doi.org/10.1001/jama.2011.369

4. Sullivan MD. Limiting the potential harms of high-dose opioid therapy: comment on "Opioid dose and drug-related mortality in patients with nonmalignant pain”. Arch Intern Med. 2011;171(7):691-3. doi:https:// doi.org/10.1001/archinternmed.2011.101

5. Compton WM, Boyle M, Wargo E. Prescription opioid abuse: Problems and responses. Prev Med. 2015;80:5-9. doi:https://doi.org/10.1016/j. ypmed.2015.04.003

6. Boslett AJ, Denham A, Hill EL, Adams MCB. Unclassified drug overdose deaths in the opioid crisis: emerging patterns of inequity. J Am Med Inform Assoc. 2019. doi:https://doi.org/10.1093/jamia/ocz050

7. Florence CS, Zhou C, Luo F, Xu L. The Economic Burden of Prescription Opioid Overdose, Abuse, and Dependence in the United States, 2013. Med Care. 2016;54(10):901-6. doi:https://doi.org/10.1097/MLR. 0000000000000625

8. Hah JM, Bateman BT, Ratliff J, Curtin C, Sun E. Chronic Opioid Use After Surgery: Implications for Perioperative Management in the Face of the Opioid Epidemic. Anesth Analg. 2017;125(5):1733-40. doi:https:// doi.org/10.1213/ANE.0000000000002458

9. Alam A, Gomes T, Zheng H, Mamdani MM, Juurlink DN, Bell CM. Long-term analgesic use after low-risk surgery: a retrospective cohort study. Arch Intern Med. 2012;172(5):425-30. doi:https://doi.org/10. 1001/archinternmed.2011.1827

10. Brummett CM, Waljee JF, Goesling J, Moser S, Lin P, Englesbe MJ, et al. New Persistent Opioid Use After Minor and Major Surgical Procedures in US Adults. JAMA Surg. 2017;152(6):e170504. doi:https://doi.org/10.1001/jamasurg.2017.0504 
11. Sun EC, Darnall BD, Baker LC, Mackey S. Incidence of and Risk Factors for Chronic Opioid Use Among Opioid-Naive Patients in the Postoperative Period. JAMA Intern Med. 2016;176(9): 1286-93. doi:https://doi.org/10. 1001/jamainternmed.2016.3298

12. Smolina K, Gladstone EJ, Rutherford K, Morgan SG. Patterns and trends in long-term opioid use for non-cancer pain in British Columbia, 2005-2012. Can J Public Health. 2016; 107(4-5):e404-e9. doi:https://doi. org/10.17269/cjph.107.5413

13. Campbell CI, Weisner C, Leresche L, Ray GT, Saunders K, Sullivan MD, et al. Age and gender trends in long-term opioid analgesic use for noncancer pain. Am J Public Health. 2010;100(12):2541-7. doi:https:// doi.org/10.2105/AJPH.2009.180646

14. Clarke H, Soneji N, Ko DT, Yun L, Wijeysundera DN. Rates and risk factors for prolonged opioid use after major surgery: population based cohort study. BMJ. 2014;348:g1251. doi:https://doi.org/10.1136/bmj. g1251

15. Brat GA, Agniel D, Beam A, Yorkgitis B, Bicket M, Homer M, et al. Postsurgical prescriptions for opioid naive patients and association with overdose and misuse: retrospective cohort study. BMJ. 2018;360:j5790. doi:https://doi.org/10.1136/bmj.j5790

16. Brannon PM. Key questions in vitamin D research. Scandinavian journal of clinical and laboratory investigation. Supplementum. 2012;243:15462. doi:https://doi.org/10.3109/00365513.2012.682895

17. Hamilton B. Vitamin D and human skeletal muscle. Scand J Med Sci Sports. 2010;20(2): 182-90. doi:https://doi.org/10.1111/j.1600-0838. 2009.01016.x

18. Plotnikoff GA, Quigley JM. Prevalence of severe hypovitaminosis D in patients with persistent, nonspecific musculoskeletal pain. Mayo Clin Proc. 2003;78(12):1463-70. doi:https://doi.org/10.4065/78.12.1463

19. McCabe PS, Pye SR, Beth JM, Lee DM, Tajar A, Bartfai G, et al. Low vitamin $\mathrm{D}$ and the risk of developing chronic widespread pain: results from the European male ageing study. BMC Musculoskelet Disord. 2016;17:32. doi:https://doi.org/10.1186/s12891-016-0881-6

20. Lee A, Chan SK, Samy W, Chiu CH, Gin T. Effect of Hypovitaminosis D on Postoperative Pain Outcomes and Short-Term Health-Related Quality of Life After Knee Arthroplasty: A Cohort Study. Medicine (Baltimore). 2015:94(42):e1812. doi:https://doi.org/10.1097/MD 0000000000001812

21. Lotfi A, Abdel-Nasser AM, Hamdy A, Omran AA, El-Rehany MA Hypovitaminosis D in female patients with chronic low back pain. Clin Rheumatol. 2007;26(11):1895-901. doi:https://doi.org/10.1007/ s10067-007-0603-4

22. Al-Allaf AW, Mole PA, Paterson CR, Pullar T. Bone health in patients with fibromyalgia. Rheumatology (Oxford). 2003;42(10):1202-6. doi:https://doi.org/10.1093/rheumatology/keg356

23. Prakash S, Rathore C, Makwana P, Dave A. Joshi H, Parekh H. Vitamin D Deficiency in Patients With Chronic Tension-Type Headache: A CaseControl Study. Headache. 2017;57(7):1096-108. doi:https://doi.org/10. 1111/head.13096

24. Glover TL, Goodin BR, Horgas AL, Kindler LL, King CD, Sibille KT, et al. Vitamin D, race, and experimental pain sensitivity in older adults with knee osteoarthritis. Arthritis Rheum. 2012;64(12):3926-35. doi:https://doi.org/10.1002/art.37687

25. von Kanel R, Muller-Hartmannsgruber V, Kokinogenis G, Egloff $\mathbf{N}$ Vitamin $\mathrm{D}$ and central hypersensitivity in patients with chronic pain. Pain Med. 2014;15(9):1609-18. doi:https://doi.org/10.1111/pme. 12454

26. Bergman P, Sperneder S, Hoijer J, Bergqvist J, Bjorkhem-Bergman L Low vitamin $D$ levels are associated with higher opioid dose in palliative cancer patients-results from an observational study in Sweden. PLoS One. 2015;10(5):e0128223. doi:https://doi.org/10.1371/journal.pone. 0128223

27. Turner MK, Hooten WM, Schmidt JE, Kerkvliet JL, Townsend CO, Bruce BK. Prevalence and clinical correlates of vitamin D inadequacy among patients with chronic pain. Pain Med. 2008;9(8):979-84. doi:https://doi.org/10.1111/j.1526-4637.2008.00415.x

28. Munns CF, Shaw N, Kiely M, Specker BL, Thacher TD, Ozono K, et al. Global Consensus Recommendations on Prevention and Management of Nutritional Rickets. J Clin Endocrinol Metab. 2016;101(2):394-415. doi:https://doi.org/10.1210/jc.2015-2175
29. Larochelle MR, Zhang F, Ross-Degnan D, Wharam JF. Rates of opioid dispensing and overdose after introduction of abuse-deterrent extendedrelease oxycodone and withdrawal of propoxyphene. JAMA Intern Med. 2015;175(6):978-87. doi:https://doi.org/10.1001/jamainternmed.2015. 0914

30. Macrae WA. Chronic pain after surgery. Br J Anaesth. 2001;87(1):88-98. doi:https://doi.org/10.1093/bja/87.1.88

31. Von Korff M, Saunders K, Thomas Ray G, Boudreau D, Campbell C, Merrill J, et al. De facto long-term opioid therapy for noncancer pain. Clin J Pain. 2008;24(6):521-7. doi:https://doi.org/10.1097/AJP. Ob013e318169d03b

32. Cepeda MS, Fife D, Ma $\mathbf{Q}$, Ryan PB. Comparison of the risks of opioid abuse or dependence between tapentadol and oxycodone: results from a cohort study. J Pain. 2013;14(10):1227-41. doi:https://doi.org/10.1016/ j.jpain.2013.05.010

33. Krieger N, Waterman PD, Spasojevic J, Li W, Maduro G, Van Wye G. Public Health Monitoring of Privilege and Deprivation With the Index of Concentration at the Extremes. Am J Public Health. 2016;106(2):256-63. doi:https://doi.org/10.2105/AJPH.2015.302955

34. Wharam JF, Lu CY, Zhang F, Callahan M, Xu X, Wallace J, et al. HighDeductible Insurance and Delay in Care for the Macrovascular Complications of Diabetes. Ann Intern Med. 2018;169(12):845-54. doi:https:// doi.org/10.7326/M17-3365

35. Holick MF, Chen TC. Vitamin D deficiency: a worldwide problem with health consequences. Am J Clin Nutr. 2008;87(4):1080S-6S.

36. Lips P. Vitamin D physiology. Prog Biophys Mol Biol. 2006;92(1):4-8. doi:https://doi.org/10.1016/j.pbiomolbio.2006.02.016

37. Holick MF. Vitamin D deficiency. N Engl J Med. 2007;357(3):266-81. doi:https://doi.org/10.1056/NEJMra070553

38. Basit A, Basit KA, Fawwad A, Shaheen F, Fatima N, Petropoulos IN, et al. Vitamin D for the treatment of painful diabetic neuropathy. BMJ Open Diabetes Res Care. 2016;4(1):e000148. doi:https://doi.org/10. 1136/bmjdrc-2015-000148

39. Shipton EE, Shipton EA. Vitamin D Deficiency and Pain: Clinical Evidence of Low Levels of Vitamin D and Supplementation in Chronic Pain States. Pain Ther. 2015;4(1):67-87. doi:https://doi.org/10.1007/ s40122-015-0036-8

40. Le Goaziou MF, Kellou N, Flori M, Perdrix C, Dupraz C, Bodier E, et al. Vitamin D supplementation for diffuse musculoskeletal pain: results of a before-and-after study. Eur J Gen Pract. 2014;20(1):3-9. doi:https://doi. org/10.3109/13814788.2013.825769

41. Yong WC, Sanguankeo A, Upala S. Effect of vitamin D supplementation in chronic widespread pain: a systematic review and meta-analysis. Clin Rheumatol. 2017. doi:https://doi.org/10.1007/s10067-017-3754-y

42. Khan QJ, Kimler BF, Reddy PS, Sharma P, Klemp JR, Nydegger JL, et al. Randomized trial of vitamin D3 to prevent worsening of musculoskeletal symptoms in women with breast cancer receiving adjuvant letrozole. The VITAL trial. Breast cancer research and treatment. 2017. https://doi.org/10.1007/s10549-017-4429-8

43. Helde-Frankling M, Hoijer J, Bergqvist $\mathbf{J}$, Bjorkhem-Bergman $\mathbf{L}$. Vitamin D supplementation to palliative cancer patients shows positive effects on pain and infections-Results from a matched case-control study. PLoS One. 2017;12(8):e0184208. doi:https://doi.org/10.1371/journal. pone.0184208

44. Kuru P, Akyuz G, Yagci I, Giray E. Hypovitaminosis D in widespread pain: its effect on pain perception, quality of life and nerve conduction studies. Rheumatol Int. 2015;35(2):315-22. doi:https://doi.org/10. 1007/s00296-014-3099-7

45. Tague SE, Clarke GL, Winter MK, McCarson KE, Wright DE, Smith PG. Vitamin D deficiency promotes skeletal muscle hypersensitivity and sensory hyperinnervation. J Neurosci. 2011;31(39):13728-38. doi:https://doi.org/10.1523/JNEUROSCI.3637-11.2011

46. Fell GL, Robinson KC, Mao J, Woolf CJ, Fisher DE. Skin betaendorphin mediates addiction to UV light. Cell. 2014;157(7):1527-34. doi:https://doi.org/10.1016/j.cell.2014.04.032

Publisher's Note: Springer Nature remains neutral with regard to jurisdictional claims in published maps and institutional affiliations. 